Memorias del VII Encuentro Nacional de Experiencias en la Enseñanza de la Biología y la Educación Ambiental y II Congreso Nacional de Investigación en la Enseñanza de la Biología

\title{
LA TRANSFORMACIÓN DEL CONTENIDO BIOLÓGICO EN LOS PRIMEROS AÑOS DE EJERCICIO DOCENTE: RETOS Y DESAFÍOS DE LA INSERCIÓN PROFESIONAL
}

\author{
Ma. Mercedes Jiménez Narváez ${ }^{1}$
}

\section{Resumen}

Enseñar temas como la célula y el sistema excretor en la escuela primaria y secundaria de nuestro país, es una situación normal y recurrente para un profesor de ciencias naturales; estos temas forman parte del currículo y de los planes de estudio del área, en las instituciones educativas públicas y privadas. Pero, ¿qué ocurre cuando un profesor principiante tiene el encargo de enseñar estos temas?, ¿qué transformación del contenido biológico realiza para compartirlo con sus estudiantes?, ¿qué condiciones institucionales y personales influyen en la toma de decisiones sobre su enseñanza y evaluación?, éstas son algunas de las inquietudes que dieron origen a una tesis doctoral ${ }^{2}$ que indaga por la configuración del conocimiento profesional del profesor, en la etapa de la carrera docente conocida como inserción profesional. Se busca describir y comprender los componentes del conocimiento del profesor desde la perspectiva del Pedagogical Content Knowledge (PCK) sugerida por el grupo de Magnusson, Krajcik y Borko (1999), y además, su relación con los factores de socialización docente propuesta por Jordell (1987). Los resultados indican que las vivencias de los primeros años de ejercicio docente, las relaciones con los estudiantes y el contexto institucional y social de la profesión, influyen de forma decisiva en los procesos de transformación del contenido a enseñar que realiza un profesor principiante.

\begin{abstract}
Abstrac
Teachingsubjects likecell andthe excretory systeminprimary and secondary schoolsin our country, is a normaland recurringnaturalscience teacher, these issuesare partof the curriculumarea institutions public and privateeducation. But whathappen when abeginning teacherhas teachingthese subjects, and what biologicalcontent transformationdone toshare with your students, and what personaland institutional conditionsinfluencedecisions abouttheir teaching and assessment?, these aresome of the concernsthat led toa doctoral thesisabouttheconfiguration ofteachers' professional knowledgeinteaching careerstageknown asteacher induction. Itseeks to describeand understandthe

1 Profesora Facultad de Educación, Universidad de Antioquia. Miembro Grupo GECEM. mjimenez@ayura.udea.edu.co

2 Jiménez Narváez, M.M. (2013). Profesor/a principiante de ciencias naturales: la configuración de su conocimiento en la inserción profesional. Facultad de Educación Universidad de Antioquia. Tesis doctoral sin publicar.
\end{abstract}


Memorias del VII Encuentro Nacional de Experiencias en la Enseñanza de la Biología y la Educación Ambiental y II Congreso Nacional de Investigación en la Enseñanza de la Biología

components ofteacher knowledgefrom the perspective ofPedagogical ContentKnowledge(PCK) groupsuggested byMagnusson, KrajcikandBorko(1999), andalsotheir relationship with theteachersocialization factorsproposed byJordell(1987).The results indicate thatthe experiencesof the firstyears of teaching, relationships with students andtheinstitutional and social contextof the professionhave a decisive influenceon the processes oftransformation ofteaching contentthatmakes abeginning teacher.

Palabras Claves: Profesor principiante; Conocimiento profesional docente; Pedagogical Content Knowledge (PCK); Socialización profesional; célula; sistema excretor.

Key Words: Beginning teacher; Teachers' professional knowledge; Pedagogical Content Knowledge (PCK); Professional socialization; Cell; Excretory system.

\section{Introducción}

En esta comunicación se presentan algunos resultados y reflexiones generadas en una tesis doctoral, que se realizó con la participación de cuatro egresados/as de un programa de formación inicial ${ }^{3}$.

Los objetivos de la tesis se concentran, en primer lugar, en comprender el proceso de inserción profesional docente y su influencia en la configuración del conocimiento profesional -especialmente del Pedagogical Content Knowledge (PCK) - del profesor/a principiante de ciencias naturales. En segundo lugar, dada la falta de información empírica que tenemos de las prácticas de nuestros egresados/as, se buscaba describir el conocimiento profesional que muestran los/as profesores/as principiantes de ciencias naturales cuando enseñan un contenido específico del área, tomando en cuenta cinco componentes del PCK: la orientación de la enseñanza de ciencias; las estrategias de enseñanza; las estrategias de evaluación; el currículo; y las ideas de los estudiantes sobre el tópico que se está enseñando. Finalmente, se esperaba que las evidencias de los estudios de caso nos permitieran analizar los aportes y limitaciones del PCK en la caracterización del conocimiento profesional del principiante, y a partir de ahí generar futuros procesos de formación e investigación.

Para alcanzar estos objetivos, se tomó como referentes teóricos, por un lado, a Magnusson, Krajcik y Borko (1999), que nos ayudan a comprender de manera general el concepto de conocimiento profesional del profesor, y del Pedagogical Content Knowledge (PCK) de profesores del área de ciencias naturales, en

\footnotetext{
${ }^{3}$ Se hace referencia a la Licenciatura en educación básica con énfasis en ciencias naturales y educación ambiental, Facultad de Educación, Universidad de Antioquia.
} 
Memorias del VII Encuentro Nacional de Experiencias en la Enseñanza de la Biología y la Educación Ambiental y II Congreso Nacional de Investigación en la Enseñanza de la Biología

particular. Además, su propuesta parte de la premisa que el PCK es un conocimiento que resulta de la transformación del conocimiento de la materia (saber disciplinar), el conocimiento pedagógico y el conocimiento del contexto, de ahí su riqueza y complejidad.

Por otro lado, y para comprender la condición de los profesores principiantes como aquellos egresados de un programa de formación inicial, que se enfrentan por primera vez al mundo laboral y profesional, se retoman las ideas de Tardif (2005) sugiriendo que estas vivencias de los profesores se inscriben en un campo de estudio conocido como Inserción Profesional. Esta perspectiva incluye dos dimensiones que interactúan simultáneamente: los procesos de búsqueda del primer empleo y la inserción como una etapa de la carrera docente. Esta distinción permite el reconocimiento de esta etapa, es diferente a la formación inicial y a la formación continua, por tanto tiene unas características y problemas distintivos. Dada la amplitud del tema y ya que, en el sistema de formación docente de nuestro país, aún no se tiene en cuenta con suficiente claridad la inserción profesional, se delimitó la indagación en la identificación de los factores que influyen en la socialización profesional de los docentes participantes, siguiendo la perspectiva de Jordell (1987), que propone cuatro factores: lo personal, el aula, lo institucional y lo social.

En esta comunicación se hará énfasis en los hallazgos de dos de los casos analizados: Oscar y Andrés. La práctica de estos recién egresados nos dan luces para pensar en los procesos de formación e investigación que se pueden generar con esta población de profesores y además, muestran algunos retos y desafíos que tenemos en el país frente a esta etapa de la carrera docente.

\section{Metodología}

La investigación se enmarcó en una perspectiva cualitativa, interpretativa y con estudios de caso. La recolección de información se realizó durante el 2010, con cuatro profesores/as principiantes voluntarios, egresados del mismo programa de formación inicial en Medellín, que trabajaban en instituciones educativas públicas (2) y privadas (2). Cada participante concedió tres entrevistas semiestructuradas; se realizó la observación y grabación de una secuencia completa de clase; y la revisión de documentos (personales e institucionales); además, con episodios de las grabaciones se realizó una entrevista de autoconfrontación. A la fecha de las entrevistas, Sara, Oscar, Andrés y Elsa ${ }^{4}$, llevaban laborando 4, 6, 18 y 24 meses respectivamente. Con el fin de ampliar la información sobre la inserción profesional (ingreso a la carrera, inducción, evaluación, periodo de prueba) se

\footnotetext{
${ }^{4}$ Pseudónimos escogidos por cada uno de los participantes y que han autorizado la publicación de estos materiales.
} 
Bio-grafia Escritos sobre la Biologia y su Enseñanza.

Edición Extra-Ordinaria. ISSN 2027-1034 P.p 740 - 750

Memorias del VII Encuentro Nacional de Experiencias en la Enseñanza de la Biología y la Educación Ambiental y II Congreso Nacional de Investigación en la Enseñanza de la Biología

realizaron entrevistas semiestructuradas a dos rectores, dos coordinadores de las instituciones educativas donde laboran los egresados/as; y una entrevista a una funcionaria de la Secretaría de Educación de Medellín.

Para esta comunicación solamente se hará referencia a los casos de Oscar y Andrés, a continuación algunas características (ver Tabla 1):

\begin{tabular}{l|c|c}
\hline \multicolumn{2}{c}{ Tabla 1. Características generales de participantes } \\
\hline Pseudónimo & Oscar & Andrés \\
\hline Edad Profesor/a & 22 & 25 \\
\hline $\begin{array}{l}\text { Experiencia docente (a julio } \\
\text { 2010) }\end{array}$ & 9 meses (3 el año 2009) & 1 año y 8 meses \\
\hline $\begin{array}{l}\text { Carácter de la Institución y } \\
\text { Jornada académica }\end{array}$ & $\begin{array}{c}\text { Privada - Mixta; 12:30 - 5:30 p.m. } \\
\text { (horario profesor 10:00-6:00) }\end{array}$ & $\begin{array}{c}\text { Pública- Mixta } \\
12: 00-6: 00 \text { p.m. }\end{array}$ \\
\hline Ubicación & Barrio Popular 1, Medellín & $\begin{array}{c}\text { Correg. de San Cristóbal, } \\
\text { Medellín }\end{array}$ \\
\hline No. horas área ciencias & 2 horas/semana & 4 horas/semana \\
naturales & 40 & 60 \\
\hline Grupo estudio & 32 & 36 \\
\hline No. estudiantes & Célula & Sistema Excretor \\
\hline Tema elegido & Miércoles y Viernes 12:30 -2:30; y & Martes 4:00 - 6:00; 3:00 - 4:00 \\
\hline Horario grabación & una Martes 12:30 - 2:30 p.m. & $6: 00$ p.m. \\
\hline No. de sesiones grabadas & 4 clases; 8horas & 6 clases; 9 horas \\
\hline
\end{tabular}

La transcripción, análisis y categorización de cada entrevista y de las secuencias de enseñanza, permitió posteriormente encontrar puntos comunes y diferentes, que nos sirven de evidencia para comprender el concepto de inserción, su relación con la manera cómo se configura el conocimiento profesional del docente y sus implicaciones tanto para la formación como para la investigación en esta línea de profesores/as de ciencias naturales.

\section{Resultados y discusión}

\section{El ingreso a la carrera, la búsqueda de empleo, la inducción y la evaluación docente}

Andrés y Oscar ingresaron a la Licenciatura por segunda opción en el examen de admisión; no obstante expresaron en las entrevistas que el trabajo en algunos cursos (psicología, didáctica, y cursos del saber específico), las conversaciones con ciertos profesores/as y la experiencia de la práctica pedagógica, les sirvieron como elementos para decidir quedarse en el programa de formación. 
Bio-grafia Escritos sobre la Biologia y su Enseñanza.

Edición Extra-Ordinaria. ISSN 2027-1034 P.p 740 - 750

Memorias del VII Encuentro Nacional de Experiencias en la Enseñanza de la Biología y la Educación Ambiental y II Congreso Nacional de Investigación en la Enseñanza de la Biología

Una vez se graduaron, cada uno optó por una ruta diferente para la búsqueda de empleo. Mientras Oscar repartió hojas de vida en instituciones educativas privadas, Andrés pudo participar en la convocatoria 2009-2010 para vincularse con el sector docente oficial. Después de ser aceptados en las instituciones educativas, sigue otro paso: la inducción. Andrés al trabajar con el Estado es convocado a una jornada de un día, donde le informan los asuntos administrativos, prestacionales, la normatividad y los procesos que siguen para su vinculación definitiva. Luego llega a la institución elegida y la rectora hace una pequeña inducción, pues además de Andrés llegan 16 profesores nuevos. Oscar a su vez, participa de una jornada general de profesores y luego una sesión con la coordinadora; al día siguiente tiene que asumir las tareas propias del inicio del año (habilitaciones del anterior, matrículas, etc.). Se puede decir que ambos reciben cierta información, a manera de inducción institucional, a cargo de los rectores y coordinadores, pero resalta que esta "bienvenida" se realiza con poco tiempo, de manera informal y les entregan a los docentes información en físico y virtual, que en su mayor parte está desactualizada.

Dado que Andrés inicia su periodo de prueba, concerta con la rectora los puntos de la evaluación de desempeño, tomando como base algunas de las sugerencias emanadas por el Ministerio de Educación Nacional, pero que no necesariamente corresponden al periodo de prueba (ver Guía $\left.N^{\circ} 10,2008\right)$. En la institución de Oscar, al final del año los profesores son evaluados bajo ciertos criterios que define la rectoría y aplicados por la coordinadora, quien finalmente define su continuidad laboral.

\section{Conocimiento profesional docente}

De acuerdo a la propuesta de Magnusson, Krajcik y Borko (1999), el Conocimiento profesional del profesor se constituye desde cuatro componentes: el pedagógico general; el disciplinar o del saber específico; el del contexto; y el pedagogical content knowledge (PCK). Frente a estos tipos de conocimiento encontramos en los dos casos, características particulares dadas por las diferencias en los aspectos personales e institucionales de Oscar y Andrés.

De manera general podemos decir que frente al conocimiento pedagógico y los asuntos relacionados con la gestión del aula (control de disciplina y manejo de grupo), Andrés tuvo mayores dificultades que Oscar, para concertar los momentos de diálogo e interacción con sus estudiantes de $6^{\circ}$, a quienes conoció a mitad del año escolar en el 2010. Para Oscar estos asuntos pasaron a un segundo plano, por la cercanía que construyó con los estudiantes de $4^{\circ}$ desde el inicio del año y también, porque pudo aprovechar algunas rutinas institucionales establecidas para el control de la disciplina, las normas en el aula y el comportamiento de los niños/as. En las clases de Andrés hay continuas interrupciones, por los mismos 
Bio-grafia Escritos sobre la Biologia y su Enseñanza.

Edición Extra-Ordinaria. ISSN 2027-1034 P.p 740 - 750

Memorias del VII Encuentro Nacional de Experiencias en la Enseñanza de la Biología y la Educación Ambiental y II Congreso Nacional de Investigación en la Enseñanza de la Biología

estudiantes y también externas, las cuales hacen que él pierda el hilo de la conversación y por ello su expresión de "qué desespero", refleja la impotencia de su acción y la frustración frente a lo que quiere lograr; su reacción es acelerar el ritmo de su voz y de la clase, mantener ocupados a los estudiantes en la copia de lo que ha escrito en el tablero o llamar al coordinador de disciplina como apoyo para controlar al grupo.

En cuanto al conocimiento disciplinar, podemos decir que tanto Oscar como Andrés dieron más indicios de cómo asumen los contenidos escolares de la biología que de la biología como una disciplina. Sus expresiones están ligadas a los recuerdos de la formación inicial, reconociendo las fortalezas del eje biológico del pensum que cursaron. No obstante, sus propias creencias sobre cómo se construye el conocimiento biológico, dan cuenta de rasgos de una perspectiva tradicional de la ciencia; así por ejemplo, la experimentación y el uso del microscopio como instrumento privilegiado para "ver" la célula, en el caso de Oscar, lleva a que toda su secuencia de enseñanza se concentre en este propósito. Para Andrés, la comprensión de la biología está dada por la lectura sistemática de textos y la memorización de términos, procedimientos que transfiere a su secuencia de enseñanza. Los dos profesores privilegian información de los libros de texto y de internet para compartir con sus estudiantes; y aunque reconocen la importancia de que el maestro esté actualizado en sus conocimientos biológicos, no cuestionan la información e imágenes que toman de estas fuentes de información. Oscar tiene algunas dificultades para trabajar el modelo teórico de ser vivo y célula; y Andrés presenta algunas confusiones entre el sistema excretor y el digestivo.

En cuanto al conocimiento del contexto, los dos profesores van construyéndolo en la medida que se relacionan con la institución, la infraestructura de la Escuela, la cercanía que van logrando con sus estudiantes, las relaciones con los colegas, administrativos y con los padres de familia. De igual forma, los profesores principiantes van construyendo sus propias ideas sobre la docencia como profesión al integrar sus propias ideas e imaginarios sobre la misma, tanto por tener referentes familiares que también son docentes, como al recordar sus experiencias como estudiantes y proyectarse ahora como profesionales. Se suma a lo anterior, las ideas que van percibiendo al incorporarse en un gremio y una cultura docente, mediada por las características de cada uno de los grupos de colegas que los reciben, donde las relaciones van desde la indiferencia hasta el compañerismo, lo cual les muestra en el camino, que su rol ha cambiado y han pasado de estudiantes de la Universidad a profesionales en ejercicio.

Ahora bien, en cuanto al Pedagogical Content Knowledge (PCK) de Oscar y Andrés, las evidencias son numerosas y particulares para cada profesor, reafirmando las características que señala Abell (2008): el PCK incluye categorías 
Bio-grafia Escritos sobre la Biologia y su Enseñanza.

Edición Extra-Ordinaria. ISSN 2027-1034 P.p 740 - 750

Memorias del VII Encuentro Nacional de Experiencias en la Enseñanza de la Biología y la Educación Ambiental y II Congreso Nacional de Investigación en la Enseñanza de la Biología

separadas de conocimiento que son aplicadas sinérgicamente a problemas de la práctica; que es dinámico (no estático); el contenido (disciplina científica) es central en el PCK; y, que el PCK implica la transformación de otros tipos de conocimiento (p. 1411).

De igual forma, las descripciones que obtuvimos de los casos nos dan insumos importantes para describir la práctica cotidiana de un profesor principiante que enseña por primera vez un tema como la célula o el sistema excretor. Los subcomponentes que permiten describir el PCK son: las estrategias de enseñanza; el currículo; las ideas de los estudiantes sobre el tópico que se está enseñando; las estrategias de evaluación; y la orientación de la enseñanza de ciencias. Para esta comunicación solo se hará referencia a los tres primeros, dado en éstos se presentaron las mayores tensiones y surgieron características en los casos de Oscar y Andrés.

En las tablas 2 y 3 , se sintetizan las actividades que los profesores principiantes realizaron en sus secuencias de enseñanza para los temas concertados y que corresponden al plan de estudio institucional, del segundo semestre académico del 2010.

\begin{tabular}{c|l}
\hline \multicolumn{2}{c}{ Tabla 2. Detalle actividades de enseñanza, tema célula, Caso Oscar, grado $4^{\circ}$} \\
\hline Fechas de grabación & Actividades de enseñanza \\
\hline Agosto 13, viernes & Identificación de ser vivo y no vivo, ejercicio del robot y ficha con dibujos \\
\hline Agosto 18, miércoles & Exposición magistral, historia de la célula y teoría celular, dictado \\
\hline Agosto 31, martes & Observación de la célula (animal y vegetal) en microscopio \\
\hline Septiembre 10, viernes & Exposición magistral y dictado partes de la célula \\
\hline
\end{tabular}

\begin{tabular}{c|l}
\hline \multicolumn{2}{c}{ Tabla 3. Detalle actividades de enseñanza, tema excreción, Caso Andrés, grado $6^{\circ}$} \\
\hline Fechas de grabación & \multicolumn{1}{c}{ Actividades de enseñanza } \\
\hline Septiembre 14 & Inicio del tema excreción y explicitación de los criterios de evaluación \\
\hline Septiembre 21 & Exposición magistral filtración, excreción y secreción \\
\hline Septiembre 28 & Exposición magistral, órganos excretores \\
\hline Octubre 5 & Taller sistema excretor (resolverlo en casa e individual) \\
\hline Octubre 19 & $\begin{array}{l}\text { Recibe los talleres sistema excretor y máquinas simples. Evaluación escrita } \\
\text { sobre las preguntas del taller. }\end{array}$ \\
\hline
\end{tabular}

Respecto a lo curricular, tanto Oscar como Andrés, organizan los contenidos a enseñar siguiendo un orden disciplinar, similar a los que se encuentran en los libros de texto para los grados $4^{\circ}$ y $6^{\circ}$, tomando información adicional en libros universitarios e internet. En la planeación se privilegian los contenidos conceptuales sobre los procedimentales y actitudinales; y se organizan tomando en consideración relaciones de conceptos de lo micro a lo macro. 
Bio-grafia Escritos sobre la Biologia y su Enseñanza.

Edición Extra-Ordinaria. ISSN 2027-1034 P.p 740 - 750

Memorias del VII Encuentro Nacional de Experiencias en la Enseñanza de la Biología y la Educación Ambiental y II Congreso Nacional de Investigación en la Enseñanza de la Biología

En cuanto a las ideas que tienen los profesores sobre el aprendizaje de los estudiantes y específicamente, sobre el aprendizaje de los conceptos en cuestión, se observa que las concepciones de Oscar tiene rasgos cercanos a la que Hollon y Anderson (1987, citados por Porlán y Rivero, 1998) llaman "aprendizaje por adquisición factual"; donde se "priman las actividades de interacción de los alumnos con los materiales y recursos didácticos frente a las de exposición y contraste de ideas"(p. 123), dando una idea de que el aprendizaje se da por simple exposición a informaciones del medio; tal vez por por ello Oscar privilegia la observación de la célula en el microscopio, en las imágenes de los libros y en la elaboración de dibujos. Por su parte Andrés, en sus clases privilegia la explicación verbal, realizando algunos dibujos en el tablero y sin otros recursos de apoyo, mostrando su fuerte creencia de que una buena explicación del tema era suficiente; esto muestra que su concepción de aprendizaje está más ligada al llamado "aprendizaje receptivo", identificado en algunos estudios del grupo de Porlán, el cual consiste en que "el profesor supone que si explica bien el tema y el alumno está atento podrá retener sin problema el contenido que se presenta" (Porlán y Rivero, 1998, p. 125).

Oscar y Andrés utilizan algunas imágenes, analogías y ejemplos para explicar en sus clases. Su uso está ligado a las concepciones sobre el aprendizaje y también, a su idea de "bajar el nivel" o "simplificar" los conceptos científicos para que sus estudiantes los puedan comprender. Si bien algunos ejemplos que toma Andrés provienen de los libros de texto, encontramos una coincidencia en estos dos casos, en tanto estas representaciones no son planeadas con anterioridad sino que "surgen" en el momento mientras hablan con sus estudiantes, pensando en las características de los contextos y de las vivencias cotidianas. De esta manera aunque algunas funcionan, lastimosamente al no planearlas con anterioridad algunas de estas representaciones son más confusas para los estudiantes o quedan inconclusas perdiendo su potencia explicativa.

Podemos decir que en estos casos, el contenido a enseñar no sufre realmente un proceso de transformación, en los términos de la propuesta de Shulman (modelo de razonamiento pedagógico y de acción); si bien, los dos profesores hacen una selección de contenidos, una selección de estrategias didácticas y algunas adaptaciones a las características de los estudiantes, éstas solo dan cuenta de una transferencia de información de los libros de texto -en los cuales los profesores depositan su confianza - hacia los cuadernos de los estudiantes.

\section{Conclusiones}

Los hallazgos de esta tesis nos dieron indicios de algunas limitaciones que tienen los profesores principiantes en ciertos componentes del PCK y en el proceso de transformación del contenido a enseñar; no obstante, lo que pudimos apreciar es 
Bio-grafia Escritos sobre la Biologia y su Enseñanza.

Edición Extra-Ordinaria. ISSN 2027-1034 P.p 740 - 750

Memorias del VII Encuentro Nacional de Experiencias en la Enseñanza de la Biología y la Educación Ambiental y II Congreso Nacional de Investigación en la Enseñanza de la Biología

que esto no se debe solamente a las relaciones que establece el profesor/a entre su práctica (pedagógica y didáctica) y el conocimiento disciplinar, sino que estas debilidades están fuertemente mixturadas con los diferentes componentes del conocimiento profesional: lo pedagógico, disciplinar, el contexto y el mismo PCK.

Si bien la perspectiva del PCK y los elementos que lo conforman, nos han servido como lente teórico y metodológico interesante para avanzar en la caracterización del conocimiento de estos profesores, consideramos que son todos los componentes del conocimiento profesional -en su conjunto e interacción-, encajando como en un mosaico, los que ayudan a comprender la experiencia del profesor que está en sus primeros años en el ejercicio docente. Por tanto, la inserción profesional representa unas condiciones y tensiones particulares en la constitución de cada profesor/a.

Fue importante encontrar evidencias para comprender algunos dilemas y dificultades que tienen los profesores entre la planeación y la enseñanza de las ciencias. Esta información es insumo para las reflexiones que se dan desde la didáctica y la pedagogía, para pensar en la planeación como un proceso creativo y constructivo que orienta al profesor, genera dinámicas diversas en el aula y puede constituirse en un elemento formativo y de investigación interesante en nuestro campo.

Un asunto preocupante que se manifestó en este recorrido, fue encontrar que los profesores principiantes no tengan procesos de inducción adecuados cuando inician su labor en las instituciones educativas. Vimos que la "bienvenida" o recibimiento, cumple una función informativa y normativa, se realiza utilizando un tiempo mínimo de la jornada, sin materiales suficientes de apoyo (o desactualizados) y a cargo del personal administrativo de turno (intuición y experiencia); estos elementos podríamos decir, son indicadores de la poca importancia que se le da a este proceso.

La falta de información sobre el contexto institucional, desencadena una serie de situaciones que pueden afectar tanto al docente nuevo/a como a la Escuela: el profesor tarda más tiempo en reconocerse como miembro de esa comunidad, y puede enfrentar más dificultades para adaptarse a los requerimientos, funciones y metas que debe cumplir, pues desconoce qué se espera de él o ella; pueden surgir malentendidos y conflictos en las vías regulares de comunicación (con estudiantes, padres de familia, colegas), generando a su vez, un ambiente de tensión, la pérdida de tiempo (que podría emplearse de forma distinta) y un desgaste (emocional y físico) para todos los involucrados. Además, la institución pierde la posibilidad de identificar con mayor claridad las fortalezas y debilidades del nuevo profesor y que éste pueda adquirir un sentido de pertenencia para luchar por los propósitos comunes que los identifican. En consecuencia se 
Memorias del VII Encuentro Nacional de Experiencias en la Enseñanza de la Biología y la Educación Ambiental y II Congreso Nacional de Investigación en la Enseñanza de la Biología

considera que, la inducción y proceso de bienvenida que se realizan en las instituciones escolares, debería pensarse como un paso necesario y de gran valor para el aprendizaje docente y además, como una oportunidad para cualificar las dinámicas escolares.

Pensar en la inserción profesional como el proceso de búsqueda de empleo y como fase de la carrera docente, debe ayudarnos a reflexionar sobre la responsabilidad de los actores implicados en esta: las Facultades de Educación que formamos maestros/as; las instituciones educativas que los reciben; la administración pública que regula la docencia como profesión y por supuesto, cada profesor/a principiante.

La propuesta incluye abrir el debate de este campo de investigación en nuestro país, motivar a la comunidad académica para que formemos equipos de trabajo, especialmente tomando en consideración la actual discusión del Ministerio de Educación Nacional para la generación de una política sobre formación de profesores; y entre otros, la generación de dispositivos de acompañamiento a egresados/as para evitar que los primeros años de ejercicio docente se conviertan en una marca negativa en su desarrollo profesional.

\section{Bibliografía}

Abell, S.K., (2008). Twenty years later: Does Pedagogical content Knowledge remain a useful idea?International Journal of Science Education. 30 (10), 1405 1416.

Hernández-Sampieri, R., Fernández-Collado, C. \& Baptista, P., (2006). Metodología de la Investigación. México: Mc Graw Hill. CD-ROM complementario, Capítulo 4. Estudio de Caso.

Jordell, K., (1987). Structural and personal influences in the socialization of beginning teachers.Teaching \& Teacher Education, 3, (3), $165-177$.

Magnusson, S., Krajcik, J. \& Borko, H., (1999). Nature, Sources and Development of Pedagogical Content Knowledge for Science Teaching. En J. Gess-Newsome \& N. Lederman (Eds.) Examining Pedagogical Content Knowledge (pp. 95 - 132). Science and Technology Education Library. Kluwer Academic Publishers.

Ministerio de Educación Nacional, (2008). Guía metodológica No. 10. Evaluación de Periodo de Prueba de Docentes y Directivos Docentes. Recuperada en 2011 en, http://www.mineducacion.gov.co/cvn/1665/articles-91079 archivo pdf.pdf 
Bio-grafia Escritos sobre la Biología y su Enseñanza.

Edición Extra-Ordinaria. ISSN 2027-1034 P.p 740 - 750

Memorias del VII Encuentro Nacional de Experiencias en la Enseñanza de la

Biología y la Educación Ambiental y II Congreso Nacional de Investigación en la Enseñanza de la Biología

Porlán, R. \& Rivero, A., (1998). El conocimiento de los profesores. Una propuesta formativa en el área de ciencias. Sevilla: Diada editora.

Tardif, M., (2005). L'Insertion professionnelle dans I'enseignement. Enjeux pédagogiques. Novembre, 1, $14-18$. 